\title{
FATORES DE RISCO ASSOCIADOS À HIPOGLICEMIA E ANÁLISE DE EVENTOS ADVERSOS EM UMA TERAPIA INTENSIVA
}

\author{
Keroulay Estebanez Roque ${ }^{1}$, Andrea Rodrigues Gomes da Silva², Mario Henrique Bravo de Almeida Santos ${ }^{3}$, \\ Enirtes Caetano Prates Melo ${ }^{4}$
}

\footnotetext{
${ }^{1}$ Doutora em Ciências. Enfermeira do Hospital Universitário Clementino Fraga Filho (HUCFF) da Universidade Federal do Rio de Janeiro (UFRJ). Rio de Janeiro, Rio de Janeiro, Brasil. E-mail: keroulay@gmail.com

${ }^{2}$ Mestre em Enfermagem. Técnico Administrativo do HUCFF/UFRJ. Rio de Janeiro, Rio de Janeiro, Brasil. E-mail: dyde25@yahoo. com.br

${ }^{3}$ Especialista em Manipulação Magistral. Farmacêutico do Instituto Nacional de Traumatologia e Ortopedia do Ministério da Saúde. Rio de Janeiro, Rio de Janeiro, Brasil. E-mail: mariohbravo@yahoo.com.br

${ }^{4}$ Doutora em Saúde Pública. Pesquisadora do Departamento de Epidemiologia e Métodos Quantitativos em Saúde da Escola Nacional de Saúde Pública Sérgio Arouca da Fundação Oswaldo Cruz. Rio de Janeiro, Rio de Janeiro, Brasil. E-mail: enirtes@fiocruz.br
}

\section{RESUMO}

Objetivos: avaliar os fatores preditivos associados à ocorrência de hipoglicemia grave e analisar os eventos adversos relacionados ao uso de insulina e hipoglicemiantes orais em pacientes internados em uma unidade de terapia intensiva.

Método: trata-se de um estudo de coorte desenvolvido em um hospital de grande porte no município do Rio de Janeiro. O processo de identificação de hipoglicemia ocorreu por meio da revisão diária e prospectiva de prontuários baseada no critério de rastreamento "glicose $50 \leq \mathrm{mg} / \mathrm{dL}^{\prime \prime}$.

Resultados: foram avaliados 355 pacientes, seguidos durante toda a internação na unidade de terapia intensiva e 16 eventos de hipoglicemia relacionados ao uso de insulina foram confirmados, ocorridos em 10 pacientes, o que correspondeu a uma incidência de 2,8\% e uma taxa de incidência de 0,6 eventos de hipoglicemia por 100 pacientes-dia. Os fatores de risco associados à hipoglicemia encontrados no estudo foram: presença de doença hepática $(\mathrm{OR}=3,06$; IC $95 \%=1,22-7,66)$, sepse $(\mathrm{OR}=3,53$; IC $95 \%=1,53-8,18)$ e ocorrência de evento adverso $(\mathrm{OR}=3,89$; IC 95\%=2,05-7,41). O aumento em um ponto no escore de gravidade APACHE $(\mathrm{OR}=1,15 ; \mathrm{IC} 95 \%=1,09-1,20)$ e a ocorrência de hipoglicemia (OR=7,46; IC 95\%=3,88-14,33) implicaram em aumento da chance de óbito na UTI.

Conclusão: este estudo enfatiza o impacto da hipoglicemia na mortalidade em terapia intensiva e os fatores preditivos para sua ocorrência, bem como analisa os eventos adversos relacionados à insulina.

DESCRITORES: Hipoglicemia. Qualidade da assistência à saúde. Segurança do paciente. Terapia intensiva. Evento adverso.

\section{FACTORES DE RIESGO ASOCIADOS A LA HIPOGLICEMIA Y ANÁLISIS DE EVENTOS ADVERSOS EN UNA TERAPIA INTENSIVA}

\section{RESUMEN}

Objetivos: evaluar los factores predictivos asociados con la ocurrencia de hipoglicemia grave y analizar los eventos adversos relacionados con el uso de insulina e hipoglucemiantes orales en pacientes internados en una unidad de terapia intensiva.

Método: se trata de un estudio de cohorte desarrollado en un hospital de gran porte en el municipio de Rio de Janeiro. El proceso de identificación de hipoglicemia ocurrió por medio de la revisión diaria y prospectiva de prontuarios y basado en el criterio de rastreo "glucosa $50 \leq \mathrm{mg} / \mathrm{dL}$ ".

Resultados: se evaluaron 355 pacientes que fueron seguidos durante toda la internación en la unidad de terapia intensiva y en 16 eventos de hipoglicemia relacionados con el uso de insulina fueron confirmados. Esto ocurrió con 10 pacientes y correspondió a una incidencia del 2,8\% y una tasa de incidencia de 0,6 eventos de hipoglicemia por cada 100 pacientes por día. Los factores de riesgo asociados a la hipoglicemia encontrados en el estudio fueron la presencia de una enfermedad hepática $(\mathrm{OR}=3,06 ; \mathrm{IC} 95 \%=1,22-7,66)$, sepsia $(\mathrm{OR}=3,53$; IC $95 \%=1,53-8,18)$ y la ocurrencia de un evento adverso (OR=3,89; IC 95\%=2,05-7,41). El aumento en un punto en el resultado de gravedad APACHE (OR=1,15; IC 95\%=1,09-1,20) y la ocurrencia de hipoglicemia $(\mathrm{OR}=7,46$; IC 95\%=3,88-14,33) implicaron en el aumento de la posibilidad de óbito en la UTI.

Conclusión: este estudio enfatiza el impacto de la hipoglicemia en la mortalidad en terapia intensiva y los factores predictivos para su ocurrencia, y también, analiza los eventos adversos relacionados con la insulina.

DESCRIPTORES: Hipoglicemia. Cualidad de la asistencia para la salud. Seguridad del paciente. Terapia intensiva. Evento adverso. 


\section{INTRODUÇÃO}

A hipoglicemia em pacientes diabéticos constitui o efeito indesejável mais comum decorrente do uso de insulina e hipoglicemiantes orais, capaz de ocasionar danos cerebrais irreversíveis, falência de órgãos/tecidos e, até mesmo, óbito. A hipoglicemia grave, com valores de glicose sanguínea abaixo de $50 \mathrm{mg} / \mathrm{dL}$, pode estar presente entre pacientes diabéticos ou não, hemodinamicamente instáveis e internados em unidades de terapia intensiva (UTIs). Também está associada à mortalidade hospitalar, apresentando impacto clínico significativo na morbidade e na qualidade de vida dos pacientes. ${ }^{1-3}$

Pacientes internados em UTIs são, particularmente, vulneráveis a ocorrência de hipoglicemia, principalmente aqueles que se encontram entubados, sedados ou incapazes de reconhecer e relatar sinais e sintomas característicos. Fatores como insuficiência renal, hipotireoidismo, comprometimento cognitivo e diminuição de mecanismos regulatórios, com redução na liberação de glucagon e adrenalina, provisão insuficiente de glicose e uso de terapia para o controle glicêmico estrito estão associados ao maior risco para hipoglicemia grave. ${ }^{4-6}$

O estudo multicêntrico Normoglycemia in Intensive Care Evaluation-Survival Using Glucose Algorithm Regulation (NICE-SUGAR) acompanhou 6026 pacientes internados em UTIs. Dos pacientes em controle glicêmico estrito (glicemia 80-110 mg/dL), 45\% apresentaram hipoglicemia moderada (glicemia 41 a $70 \mathrm{mg} / \mathrm{dL}$ ) e 3,7\% hipoglicemia severa (glicemia menor ou igual $40 \mathrm{mg} / \mathrm{dL}$ ); houve associação entre ocorrência de hipoglicemia e óbito. No grupo sem infusão venosa de insulina (tratamento convencional) verificou-se o aumento de hipoglicemia moderada (15,8\%) e hipoglicemia severa $(0,5 \%) .{ }^{4}$ Esses achados estão reiterados em outros estudos. ${ }^{7-9}$

Em um estudo de coorte de pacientes internados em enfermarias de um hospital universitário, 10,5\% do grupo apresentou pelo menos um episódio de hipoglicemia, sendo que $51,2 \%$ dos eventos de hipoglicemia classificados como espontâneos (hipoglicemias não relacionadas a medicamentos). ${ }^{2} \mathrm{~A}$ ocorrência de hipoglicemia, entre pacientes diabéticos ou não implica no aumento de risco para o óbito $(\mathrm{RR}=1,67 ; \mathrm{IC} 95 \%=1,33-2,09)$. Destaca-se que apenas a hipoglicemia espontânea esteve associada à mortalidade hospitalar $(\mathrm{RR}=2,84$; IC $95 \%=2,14-3,76){ }^{2}$

A variabilidade glicêmica, acompanhada por episódios de hipoglicemia, reflete progressão de complicações do diabetes, bem como a piora da morbidade de pacientes internados em terapia intensiva. ${ }^{10-11}$ A descontinuidade no monitoramento de níveis glicêmicos pode contribuir para a alta incidência de hipoglicemia, aumentando a variabilidade da glicemia em pacientes graves. ${ }^{10,12}$

Episódio de hipoglicemia pode produzir dano ou lesão no paciente atribuído ao cuidado, comprometendo a qualidade da assistência. A avaliação constante e o monitoramento cuidadoso da glicemia pode contribuir para a redução de eventos adversos (EA) relacionados ao uso de insulina e hipoglicemiantes orais ocorridos no ambiente hospitalar, ${ }^{13}$ A notificação espontânea, a revisão de prontuários e a observação direta representam os principais métodos para a avaliação de eventos. ${ }^{13-14}$ De acordo com a Organização Mundial de Saúde (OMS), o EA é um incidente que resultou em dano desnecessário ao paciente. ${ }^{15}$

No Brasil, quatro importantes estudos identificaram a ocorrência de hipoglicemia relacionada ao uso de glibenclamida, metformina e insulina pelo método de revisão retrospectiva de prontuários utilizando o critério de rastreamento "glicemia < $50 \mathrm{mg} / \mathrm{dL}$ ". ${ }^{16-19}$ Nestes estudos, a incidência de hipoglicemia relacionada a medicamentos varia de 0,4 a 4,5 por 100 pacientes internados. ${ }^{16-19}$

Este estudo teve como objetivo avaliar os fatores preditivos associados à ocorrência de hipoglicemia grave e analisar os eventos adversos relacionados ao uso de insulina e hipoglicemiantes orais em pacientes internados em uma UTI de um hospital no município do Rio de Janeiro.

\section{MÉTODO}

Trata-se de um estudo do tipo coorte concorrente de pacientes internados em uma UTI de um hospital público de grande porte localizado no município do Rio de Janeiro. ${ }^{20}$ Baseou-se em adaptação de método proposto pelo Institute for Healthcare Improvement (IHI) e envolveu um processo de revisão de prontuários apoiado em 17 critérios de rastreamento. ${ }^{14}$

Dos rastreadores propostos pelo IHI, foi empregado 1 relacionado à assistência à saúde ("úlcera por pressão"), 4 à terapia intensiva ("caso de pneumonia", "readmissão na terapia intensiva", "complicações de procedimentos na unidade" e "entubação/reentubação") e 12 ao uso de medicamentos ("cultura positiva para Clostridium difficile", "PTT $>100$ segundos", "INR $>6$ ", " ureia aumentada ou creatinina $>2$ vezes o valor basal", " glicose $\leq 50$ $\mathrm{mg} / \mathrm{dL}$ ", "uso de vitamina $\mathrm{K}$ ", "uso de antialérgicos", "uso de flumazenil", "uso de naloxona", "uso de antieméticos", "sedação excessiva/hipotensão", "suspensão abrupta de medicamento"). ${ }^{14}$ 
A pesquisa foi realizada em um hospital terciário, referência para a alta complexidade, que desenvolve ações de ensino e pesquisa. A coorte foi formada por pacientes maiores de 18 anos admitidos no período de agosto de 2011 a julho de 2012, seguidos a partir da internação na UTI até a alta da unidade, por transferência para a enfermaria ou óbito. Foram excluídos do estudo pacientes obstétricas e internações na UTI por tempo inferior a 6 horas.

Pacientes foram monitorados para identificação de incidentes relacionados à segurança baseados na utilização dos critérios de rastreamento do IHI. ${ }^{14}$ Dos 378 pacientes internados no período, 355 foram considerados elegíveis. Não houve perdas ao longo do seguimento. No período do estudo, 27 pacientes foram reinternados.

O desfecho de interesse (hipoglicemia grave) foi avaliado pelo menos duas vezes ao dia ao longo de toda a internação. O processo de identificação de eventos obedeceu a três etapas. A primeira, realizada por duas enfermeiras, envolveu revisão diária e prospectiva de prontuários. A presença do critério de rastreamento "glicose $\leq 50 \mathrm{mg} / \mathrm{dL}^{\prime \prime 14}$ permitiu a identificação de eventos de hipoglicemia associados ao uso de insulina ou hipoglicemiantes orais, considerados evento adverso relacionado à medicamento (EAM).

Na segunda etapa foi realizada revisão do prontuário para a identificação de evidências clínicas e estabelecimento da cadeia causal de um possível incidente. Nos casos em que o registro em prontuário não foi suficiente, informações complementares foram extraídas de fontes diversas (análise de documentos adicionais, relato de pacientes, consulta às equipes médica, de enfermagem, de fisioterapia e residentes).

$\mathrm{Na}$ terceira etapa, por meio de estratégia de consenso, um médico, um farmacêutico e duas enfermeiras confirmaram a ocorrência de EAM, decorrente de hipoglicemia associada ao uso de insulina e hipoglicemiante oral.

Para ojulgamento e estabelecimento da relação de causalidade de EAMs foi utilizado o algoritmo de Naranjo. ${ }^{21}$ Divergências foram resolvidas durante as reuniões de consenso a partir do julgamento clínico. Foram considerados apenas os eventos de hipoglicemia ocorridos na terapia intensiva.

O perfil de gravidade dos pacientes foi estabelecido por meio do Índice de Comorbidade de Charlson (ICC) e do Acute Physiology and Chronic Health disease Classification II (APACHE). ${ }^{22}$ O ICC foi calculado segundo o algoritmo desenvolvido por Quan et al.,22 adaptado para a Classificação Internacional das Doenças, décima revisão (CID-10).
As covariáveis analisadas foram relacionadas às características sóciodemográficas (idade, sexo, raça/ cor, escolaridade); gravidade do paciente (avaliada pelo ICC e APACHE); número de comorbidades; presença de diabetes, doença renal, doença hepática ou sepse; tipo de admissão (clínico, cirúrgico); tempo de internação na enfermaria; tempo de internação na UTI; número de medicamentos utilizados; uso de insulina; ocorrência de EA e mortalidade na UTI.

Os testes $t$ de Student para as amostras independentes, qui-quadrado com a correção de Yates e exato de Fisher foram aplicados para a comparação de médias e entre as variáveis categóricas. Os resultados foram considerados estatisticamente significantes para um valor de $\mathrm{p}<0,05$.

A associação entre a ocorrência de EA e a variável resposta (hipoglicemia grave na UTI) foi avaliada por meio de modelos de regressão logística múltipla que estimaram a razão de chances. Com a finalidade de avaliar a associação de interesse, foram analisados modelos ajustados por sexo, idade (em anos), doença renal, doença do fígado, sepse, uso de insulina e ocorrência de EA.

Outro desfecho avaliado foi a associação entre a presença de hipoglicemia e a ocorrência de óbito na terapia intensiva, ajustado o risco pelas variáveis sexo, idade e APACHE, por meio de modelos de regressão logística múltipla. Utilizou-se o nível de significância de 0,05. A adequação do modelo final foi verificada a partir dos testes de Deviance, Hosmer-Lemeshow e Pearson. As análises estatísticas foram realizadas por meio do programa de domínio público R3.1 (R Foundation for Statistical Computing).

O presente estudo foi aprovado pelo Comitê de Ética e Pesquisa do hospital estudado sob o parecer $\mathrm{n}$ 171/10 e CAAE 20268713.2.0000.5257 e 52434716.0.00005240, atendendo à Resolução 466/12 do Conselho Nacional de Saúde. Não houve conflito de interesse no estudo.

\section{RESULTADOS}

A coorte foi formada por 355 pacientes e identificados 120 eventos de hipoglicemia grave. Foram detectados 4129 rastreadores em 311 pacientes com potenciais EAs por meio de revisão diária e prospectiva de prontuários e notificação voluntária estimulada. A idade média dos pacientes foi de 60,18 anos (DP 17,92); a maioria apresentou gravidade elevada, considerando o índice de Charlson (88,7\%), importante preditor de mortalidade (Tabela 1).

A presença de doença renal severa ou moderada, doença crônica do fígado e cirrose, doença do 
fígado severa ou moderada e metástase estiveram relacionadas à ocorrência de hipoglicemia (valor de $\mathrm{p} \leq 0,01)$. O índice de APACHE, importante pre- ditor para readmissão, mostrou-se relacionado aos episódios de hipoglicemia na população estudada (Tabela 1).

Tabela 1 - Características sociodemográficas e padrão de gravidade de pacientes internados na unidade de terapia intensiva, segundo a ocorrência de hipoglicemia. Rio de Janeiro, RJ, Brasil, 2011-2012 (n=355)

\begin{tabular}{|c|c|c|c|c|}
\hline Características & $\begin{array}{c}\text { Sem } \\
\text { hipoglicemia } \\
\text { n (\%) }\end{array}$ & $\begin{array}{c}\text { Com hipoglicemia } \\
\text { n (\%) }\end{array}$ & $\begin{array}{c}\text { Total } \\
\mathbf{n}\end{array}$ & $\begin{array}{c}\text { Valor de } \\
\qquad \mathrm{p}^{*}\end{array}$ \\
\hline$\overline{\text { Idade média [anos (DP)] }}$ & $60,01(18,13)$ & $60,92(17,10)$ & $60,18(17,92)$ & 0,709 \\
\hline \multicolumn{5}{|l|}{ Sexo } \\
\hline Feminino & $155(53,63)$ & $34(51,51)$ & 189 & \multirow[t]{2}{*}{0,862} \\
\hline Masculino & $134(46,37)$ & $32(48,49)$ & 166 & \\
\hline \multicolumn{5}{|l|}{ Raça/cor } \\
\hline Preto/negra & $39(14,18)$ & $7(10,77)$ & 46 & \multirow[t]{3}{*}{0,541} \\
\hline Parda & $87(31,63)$ & $18(27,69)$ & 105 & \\
\hline Branca & $149(54,19)$ & $40(61,54)$ & 189 & \\
\hline \multicolumn{5}{|l|}{ ICC [pontuação] } \\
\hline 0 & $63(21,80)$ & $8(12,12)$ & 71 & \multirow[t]{2}{*}{0,108} \\
\hline$\geq 1$ & $226(78,20)$ & $58(87,88)$ & 284 & \\
\hline Apache médio [pontuação (DP)] & $13,22(7,04)$ & $17,39(6,85)$ & $14,00(7,07)$ & $0,000^{*}$ \\
\hline $\begin{array}{l}\text { Média do número de } \\
\text { comorbidade (DP) }\end{array}$ & $1,97(1,72)$ & $2,21(1,54)$ & $2,01(1,69)$ & 0,229 \\
\hline Diabetes & $57(19,72)$ & $18(27,27)$ & 75 & 0,230 \\
\hline Doença renal & $19(6,57)$ & $9(13,63)$ & 28 & 0,095 \\
\hline Doença hepática & $19(6,57)$ & $10(15,15)$ & 29 & $0,040^{*}$ \\
\hline Sepse & $19(6,57)$ & $13(19,69)$ & 32 & $0,001^{*}$ \\
\hline \multicolumn{5}{|l|}{ Tipo de admissão } \\
\hline Clínico & $58(20,70)$ & $31(46,97)$ & 89 & \multirow[t]{2}{*}{$0,000^{*}$} \\
\hline Cirúrgico & $231(79,30)$ & $35(53,03)$ & 266 & \\
\hline $\begin{array}{l}\text { Tempo médio de internação na } \\
\text { enfermaria [dias (DP)] }\end{array}$ & $9,83(18,63)$ & $10,03(12,16)$ & $9,87(17,59)$ & 0,915 \\
\hline $\begin{array}{l}\text { Tempo médio de internação na } \\
\text { UTI [dias (DP)] }\end{array}$ & $8,06(12,66)$ & $17,55(15,12)$ & $9,83(13,64)$ & $0,000^{*}$ \\
\hline \multicolumn{5}{|l|}{ Número de medicamentos } \\
\hline $1-4$ & $47(16,26)$ & $1(1,51)$ & 48 & \multirow[t]{2}{*}{$0,003^{*}$} \\
\hline 5 ou mais & $242(83,74)$ & $65(98,49)$ & 307 & \\
\hline \multicolumn{5}{|l|}{ Uso de insulina } \\
\hline Não & $156(53,98)$ & $21(31,82)$ & 177 & \multirow[t]{2}{*}{$0,001^{*}$} \\
\hline Sim & $133(46,02)$ & $45(68,18)$ & 112 & \\
\hline \multicolumn{5}{|l|}{ Ocorrência de EA } \\
\hline Não & $216(74,75)$ & $27(40,90)$ & 243 & \multirow[t]{2}{*}{$0,000^{*}$} \\
\hline Sim & $73(25,25)$ & $39(59,10)$ & 112 & \\
\hline \multicolumn{5}{|l|}{ Mortalidade na UTI } \\
\hline Não & $246(85,12)$ & $26(39,40)$ & 272 & \multirow[t]{2}{*}{$0,000^{*}$} \\
\hline Sim & $43(14,88)$ & $40(60,60)$ & 83 & \\
\hline
\end{tabular}

DP: desvio padrão; ICC: Índice de Comorbidade de Charlson; EA: evento adverso; UTI: Unidade de Terapia Intensiva; *Qui-quadrado e teste $t$ de Student

Dos pacientes, $18,6 \%$ apresentaram hipoglicemia (66 eventos em 355 pacientes; IC 95\%=14,5-22,6) e uma taxa de incidência de 3,5 por 100 pacientes-dia no período (120 de 3448 pacientes-dia). A maioria 
dos casos de hipoglicemia foi internada na terapia intensiva por motivo cirúrgico $(53,0 \%)$. O tempo médio de internação na enfermaria foi de 10 dias (desvio-padrão (DP) 12,2) e na UTI, de 17,5 dias (DP 15,1), variando de 1 a 248 dias (Tabela 1).

O número de medicamentos esteve diretamente relacionado à ocorrência de hipoglicemia grave. Dos pacientes que apresentaram hipoglicemia, $98,5 \%$ faziam uso de 5 medicamentos ou mais. Observou-se diferença estatisticamente significante entre a ocorrência de hipoglicemia e a presença de um EA categorizado em diferentes grupos, conforme proposto pela $\mathrm{OMS}^{15}$ (valor de $\left.\mathrm{p} \leq 0,000\right)$ (Tabela 1 ).

Considerando a hipoglicemia como evento de interesse, foram ajustados seis modelos na análise de regressão logística múltipla (Tabela 2). No primeiro modelo, foram incluídas as variáveis consideradas para o ajuste de gravidade (sexo e idade). Dos fatores associados, o sexo, a idade e a presença de doença renal, mesmo sem significância estatística, foram mantidos em todos os modelos em decorrência do seu significado clínico e epidemiológico.

No modelo final, foram incluídas as variáveis de ajuste de gravidade, a presença de doença hepática e sepse, o uso de insulina e a ocorrência de EA (modelo 6). A presença de doença hepática $(\mathrm{OR}=3,06$; IC 95\%=1,22-7,66), de sepse $(\mathrm{OR}=3,53$; IC $95 \%=1,53-8,18)$ e a ocorrência de EA (OR=3,89; IC $95 \%=2,05-7,41)$ implicaram em aumento da chance de hipoglicemia na terapia intensiva (Tabela 2).

Tabela 2 - Associação entre características relacionadas ao paciente e a ocorrência de hipoglicemia grave. Rio de Janeiro, RJ, Brasil, 2011-2012. ( $\mathrm{n}=355)$

\begin{tabular}{lcccccc}
\hline & $\begin{array}{c}\text { Modelo 1* } \\
\text { OR (IC 95\%) }\end{array}$ & $\begin{array}{c}\text { Modelo 2* } \\
\text { OR (IC 95\%) }\end{array}$ & $\begin{array}{c}\text { Modelo 3* } \\
\text { OR (IC 95\%) }\end{array}$ & $\begin{array}{c}\text { Modelo 4* } \\
\text { OR (IC 95\%) }\end{array}$ & $\begin{array}{c}\text { Modelo 5* } \\
\text { OR (IC 95\%) }\end{array}$ & $\begin{array}{c}\text { Modelo 6* } \\
\text { OR (IC 95\%) }\end{array}$ \\
\hline Sexo masculino & $1,08(0,63-1,85)$ & $1,10(0,64-1,88)$ & $1,03(0,59-1,78)$ & $1,03(0,59-1,79)$ & $1,04(0,59-1,82)$ & $1,16(0,65-2,08)$ \\
Idade & $1,00(0,98-1,01)$ & $1,00(0,98-1,01)$ & $1,00(0,98-1,02)$ & $1,00(0,98-1,02)$ & $1,00(0,98-1,01)$ & $1,00(0,98-1,01)$ \\
Doença renal & - & $2,26(0,97-5,26)$ & $2,32(0,98-5,44)$ & $2,07(0,86-4,98)$ & $1,96(0,81-4,76)$ & $1,53(0,60-3,90)$ \\
Doença hepática & - & - & $2,68(1,15-6,20)$ & $3,00(1,28-7,02)$ & $2,45(1,03-5,83)$ & $3,06(1,22-7,66)$ \\
Sepse & - & - & - & $3,59(1,64-7,85)$ & $3,32(1,50-7,38)$ & $3,53(1,53-8,18)$ \\
Uso de insulina & - & - & - & - & $2,16(1,20-3,89)$ & $1,25(0,64-2,42)$ \\
Presença de EA & - & - & - & - & - & $3,89(2,05-7,41)$ \\
\hline
\end{tabular}

OR: Odds Ratio; IC: intervalo de confiança; EA: evento adverso; * Modelo de regressão logística múltipla

Dos 83 óbitos ocorridos durante o período de internação, 40 casos $(60,60 \%)$ apresentaram hipoglicemia durante a internação na terapia intensiva; ocorreram em média 1,7 episódios de hipoglicemia por paciente (70 de 40$)$. Observou-se diferença estatisticamente significante entre a ocorrência de hipoglicemia e óbito (valor de $\mathrm{p} \leq 0,000$ ) (Tabela 1 ).

Para estimar o efeito da associação entre a presença de hipoglicemia e a ocorrência de óbito, foram ajustados dois modelos de regressão logística múltipla. No segundo modelo, foram incluídas as variáveis de ajuste de risco do paciente e ocorrência de hipoglicemia. Cada aumento em um ponto no escore de gravidade APACHE (OR=1,15; IC 95\%=1,09$1,20)$ e a ocorrência de hipoglicemia grave $(\mathrm{OR}=7,46$; IC $95 \%=3,88-14,33$ ) implicaram em aumento da chance de óbito na UTI (Tabela 3).

Tabela 3 - Associação entre a presença de hipoglicemia grave e a ocorrência de óbito. Rio de Janeiro, RJ, Brasil, 2011-2012. $(n=355)$

\begin{tabular}{lcc}
\hline & $\begin{array}{c}\text { Modelo 1* } \\
\text { OR (IC 95\%) }\end{array}$ & $\begin{array}{c}\text { Modelo 2* } \\
\text { OR (IC 95\%) }\end{array}$ \\
\hline Sexo masculino & $1,29(0,75-2,24)$ & $1,26(0,70-2,28)$ \\
Idade & $1,00(0,99-1,02)$ & $1,00(0,99-1,02)$ \\
Apache & $1,16(1,111-21)$ & $1,15(1,09-1,20)$ \\
Hipoglicemia & - & $7,46(3,88-14,33)$ \\
\hline
\end{tabular}

OR: Odds Ratio; IC: intervalo de confiança; * Modelo de regressão logística múltipla 
A maioria dos casos de hipoglicemia não se mostrou relacionada ao uso de hipoglicemiantes orais e insulinas do tipo regular e NPH $(n=103)$. Do total de eventos investigados, 16 episódios de hipoglicemia em 10 casos foram associados a medicamentos (insulina regular e NPH) (Tabela 4) e, um caso esteve relacionado à interrupção de dieta enteral. A proporção de pacientes com eventos de hipoglicemia associado ao uso de insulina foi de $2,8 \%$ (10 de 355 ; IC $95 \%=1,28-4,90)$ e a taxa de incidência de eventos associados à insulina foi de 0,5 por 100 pacientes-dia (16 de 3448 pacientes-dia).
Dos 10 casos de hipoglicemia grave associados ao uso de insulina, 4 casos estiveram relacionados à insulina NPH administrada por via subcutânea (SC) (Tabela 4, casos designados como $\mathrm{n}^{\circ} 18,189,212$ e 261), 3 casos associados à combinação de insulina regular IV e NPH SC (Tabela 4; pacientes designados como casos $n^{\circ}$ 90, 194 e 328), 2 ocorreram devido ao uso de insulina venosa ( $\operatorname{casos} n^{\circ} 137$ e 151) e um caso mostrou-se relacionado ao uso de insulina regular por via SC (Tabela 4 casos $n^{\circ} 72$ ). Dos pacientes, $70 \%$ eram casos previamente diagnosticados como diabetes.

Tabela 4 - Casos de eventos adversos relacionados ao uso de insulina em pacientes internados na unidade de terapia intensiva. Rio de Janeiro, RJ, Brasil, 2011-2012.(n=10)

\section{Características do paciente (idade, sexo e diagnóstico)}

\section{n de eventos de hipoglicemia}

\section{Dia do evento de hipoglicemia}

\section{Medicamento suspeito}

\begin{tabular}{|c|c|c|c|}
\hline $\begin{array}{l}\text { Caso n }^{\circ} 18-52 \text { anos, feminino, hipertensão } \\
\text { arterial sistêmica e diabetes melito }\end{array}$ & 1 & $21^{\circ}$ dia & Insulina NPH (SC) \\
\hline $\begin{array}{l}\text { Caso n }{ }^{\circ} 189 \text { - } 79 \text { anos, masculino, neoplasia } \\
\text { de cólon }\end{array}$ & 1 & $28^{\circ}$ dia & Insulina NPH (SC) \\
\hline Caso $\mathrm{n}^{\mathrm{o}} 212$ - 45 anos, feminino, asma & 1 & $18^{\circ}$ dia & Insulina NPH (SC) \\
\hline $\begin{array}{l}\text { Caso no } 261 \text { - } 67 \text { anos, feminino, hipertensão } \\
\text { arterial sistêmica e diabetes melito }\end{array}$ & 2 & $18^{\circ}$ e $29^{\circ}$ dias & Insulina NPH (SC) \\
\hline $\begin{array}{l}\text { Caso no } 90 \text { - } 63 \text { anos, masculino, colecistite } \\
\text { enfisematosa, diabetes melito e insuficiência } \\
\text { renal crônica }\end{array}$ & 2 & $15^{\circ}$ e $18^{\circ}$ dias & $\begin{array}{l}\text { Insulina regular (IV e } \\
\text { SC) e NPH (SC) }\end{array}$ \\
\hline $\begin{array}{l}\text { Caso } n^{0} 194 \text { - } 20 \text { anos, masculino, } \\
\text { cetoacidose diabética e insuficiência renal } \\
\text { crônica }\end{array}$ & 4 & $9^{\circ}, 11^{\circ}, 15^{\circ}$ e $16^{\circ}$ dias & $\begin{array}{c}\text { Insulina regular (IV) e } \\
\mathrm{NPH}(\mathrm{SC})\end{array}$ \\
\hline $\begin{array}{l}\text { Caso no } 328 \text { - } 75 \text { anos, feminino, edema } \\
\text { agudo de pulmão, hipertensão arterial } \\
\text { sistêmica e diabetes melito }\end{array}$ & 2 & $7^{\circ}$ e $8^{\circ}$ dias & $\begin{array}{c}\text { Insulina regular (SC) e } \\
\mathrm{NPH}(\mathrm{SC})\end{array}$ \\
\hline $\begin{array}{l}\text { Caso } n^{\circ} 137 \text { - } 33 \text { anos, feminino, neoplasia } \\
\text { de fígado e pâncreas }\end{array}$ & 1 & $11^{\circ}$ dia & Insulina regular (IV) \\
\hline $\begin{array}{l}\text { Caso } \mathrm{n}^{\circ} 151-45 \text { anos, feminino, diabtetes } \\
\text { melito, hipotireoidismo e hepatite } C\end{array}$ & 1 & $5^{\circ}$ dia & Insulina regular (IV) \\
\hline $\begin{array}{l}\text { Caso } \mathrm{n}^{\mathrm{o}} 72 \text { - } 77 \text { anos, masculino, hipertensão } \\
\text { arterial sistêmica, diabetes melito e } \\
\text { insuficiência renal crônica }\end{array}$ & 1 & $21^{\circ}$ dia & Insulina regular (SC) \\
\hline Total & 16 & - & - \\
\hline
\end{tabular}

SC: subcutâneo; IV: intravenosa

A glicemia de pacientes que apresentaram hipoglicemia grave associada ao uso de insulina variou de 16 a $449 \mathrm{mg} / \mathrm{dL}$. Na figura 1, apresenta-se a evolução dos menores valores de glicemia observados diariamente em cada paciente durante o período de internação na UTI (até o $30^{\circ}$ dia). Com respeito à variação da resposta média ao longo da avaliação, não foi observada regularidade no padrão de comportamento da glicemia capilar. No $18^{\circ}$ e $29^{\circ}$ dia de internação, o caso $n^{\circ} 261$ (Tabela 4) apresentou dois episódios de hipoglicemia relacionada ao uso de NPH por via SC (49 mg/dL e $46 \mathrm{mg} / \mathrm{dL})$. Cabe 
destacar um dos casos que apresentou quatro eventos consecutivos de hipoglicemia grave $(45 \mathrm{mg} / \mathrm{dL}, 49$ $\mathrm{mg} / \mathrm{dL}, 45 \mathrm{mg} / \mathrm{dL}$ e $40 \mathrm{mg} / \mathrm{dL})$ no $9^{\circ}, 11^{\circ}, 15^{\circ}$ e $16^{\circ}$ dias de internação na UTI, respectivamente (caso identificado $\mathrm{n}^{\mathrm{O}} 194$ ); todos os eventos estiveram relacionados ao uso de insulina regular venosa e NPH.

Figura 1 - Evolução da menor glicemia de cada paciente internado na unidade de terapia intensiva, segundo a ocorrência de hipoglicemia relacionada ao uso de insulina e hipoglicemiantes orais. Rio de Janeiro, RJ, Brasil, 2011-2012.(n=10)

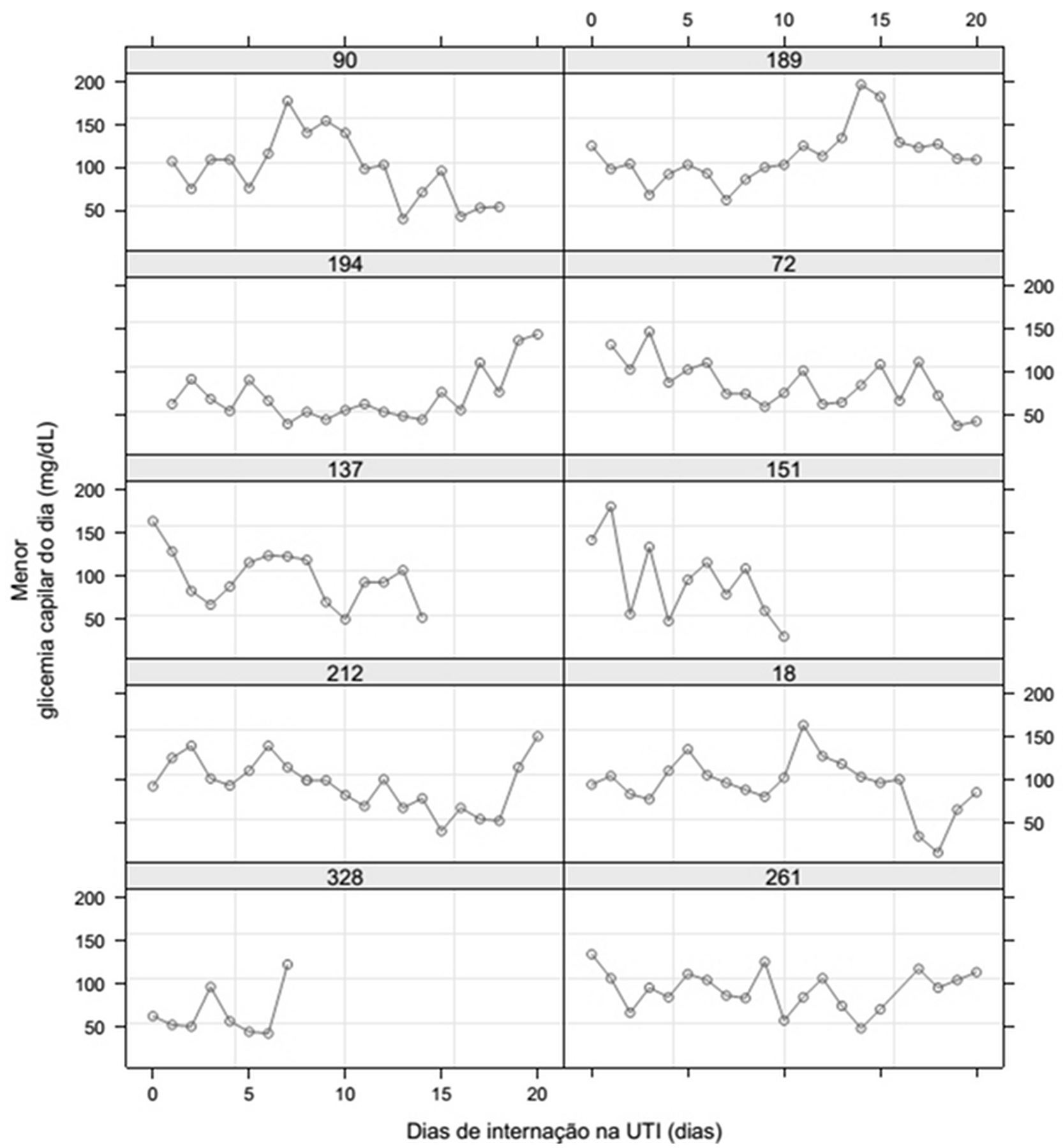




\section{DISCUSSÃO}

$\mathrm{Na}$ terapia intensiva, a hipoglicemia é um evento grave em paciente com e sem diabetes e a proporção de ocorrência varia de 3,7 a 10,0 por 100 pacientes admitidos nas UTIs. ${ }^{4,-6}$ Neste estudo, a proporção de hipoglicemia foi elevada, sendo superior às estimativas de estudos internacionais., ${ }^{4,6-7}$ Cabe destacar que a definição de hipoglicemia adotada neste estudo (glicose $\leq 50 \mathrm{mg} / \mathrm{dL}$ ) e preconizada pelo $\mathrm{IHI}^{14}$ diverge do ponto de corte proposto em alguns estudos.

Os resultados destes estudos podem ser influenciados pela forma de operacionalizar as medidas de interesse. A relevância dos eventos de hipoglicemia e sua relação com o uso de hipoglicemiantes orais e insulina impõe a padronização de critérios em estudos de monitoramento de incidentes relacionados à segurança do paciente com vistas a permitir sua comparabilidade no tempo e entre grupos.

Acredita-se que a avaliação da glicemia ser realizada pelo menos duas vezes ao dia ao longo de toda a internação favoreceu a identificação de maior proporção de hipoglicemia grave. Adicionalmente, a racionalidade empregada na identificação e no processo de confirmação de um episódio de hipoglicemia pode explicar grande parte da variabilidade das estimativas deste evento.

Os episódios de hipoglicemia se mostraram associados à ocorrência de óbito na terapia intensiva, reafirmando o importante impacto clínico na morbidade e na qualidade de vida de pacientes, já destacada em outros estudos. ${ }^{4-7,23}$ Estudos de NICESUGAR, ${ }^{4}$ Todi \& Bhattacharya, ${ }^{5}$ Kalfon et al., ${ }^{6}$ Hermanides et al., ${ }^{7}$ Garg et al., ${ }^{23}$ e observaram associação estatisticamente significativa entre hipoglicemia e mortalidade. O estudo de Boucai et al. $^{2}$ destaca que a ocorrência de hipoglicemia espontânea aumenta o risco de morte hospitalar; no entanto, a hipoglicemia relacionada ao medicamento não foi fator de risco para mortalidade. A labilidade glicêmica e as dificuldades na manutenção de controle glicêmico satisfatório devem ser consideradas em paciente graves a fim de reduzir à ocorrência de dano decorrente da assistência prestada.

A análise de regressão logística mostrou que doença hepática, sepse e ocorrência de EA são fatores relacionados ao aumento da chance de hipoglicemia. Estudos descrevem que o sexo feminino, a gravidade definida pelo escore de APACHE, o suporte de ventilação mecânica, o uso de glicocorticoide, a necessidade de hemodiálise, a falência cardíaca, a terapêutica de controle glicêmico estrito e o tempo de internação na terapia intensiva são fatores de risco para hipoglicemia. ${ }^{4,24}$ Identificar os fatores de risco relacionados à hipoglicemia permite orientar os profissionais de enfermagem na melhoria de questões relacionadas à segurança no controle glicêmico e à qualidade do cuidado prestado e, ainda, intervir no processo assistencial a fim de criar estratégias e medidas preventivas visando redução de dano.

No presente estudo, foram identificadas todas as glicemias com valores iguais ou inferiores a $50 \mathrm{mg} / \mathrm{dL}$; todavia, considerou-se EA apenas os episódios relacionados ao uso de medicamentos (insulina regular e NPH). Eventos de hipoglicemia que atendiam ao ponto de corte estabelecido e que não estavam relacionados ao uso de medicamentos (suporte calórico insuficiente, jejum prolongado, entre outras) não foram submetidos à avaliação do consenso de especialistas.

Sabe-se, ainda, que a internação na terapia intensiva implica em mudanças no seu padrão alimentar cotidiano (frequência, quantidade e tipo de dieta) e no uso concomitante de vários medicamentos, fatores que podem influenciar o nível sérico de glicose em pacientes críticos. ${ }^{25}$ Acredita-se que a reavaliação destes casos permitirá a detecção de novos eventos, baseados em outros conhecimentos, ou seja, causas não relacionadas aos medicamentos.

É importante destacar que a redução da infusão de glicose em hidratação venosa ou dieta parenteral e a interrupção da dieta enteral são fatores que aumentam o risco para ocorrência de hipoglicemia. ${ }^{25}$ Há situações em que a dieta enteral é interrompida inesperadamente para realização de exames ou administração de medicações, devendo haver prescrição de soro glicosado e mudança ou suspensão do esquema de insulina durante a pausa alimentar.

Dos 10 pacientes com hipoglicemia associada a medicamento, metade dos casos foi devido à combinação de insulina regular intravenosa (IV) e NPH ou regular por via $S C$ ( $n=11$ episódios). A insulina está entre os cinco medicamentos que causam mais danos a pacientes adultos e pediátricos, considerada um medicamento potencialmente perigoso pelo risco elevado de lesão ao paciente quando existe falha no processo de utilização. ${ }^{26-27}$ A principal complicação da infusão contínua de insulina é a ocorrência de hipoglicemia severa $(<40 \mathrm{mg} / \mathrm{dL})$, aumentando em seis vezes o risco de desenvolver o evento. ${ }^{28}$

O manejo clínico de valores glicêmicos, no entanto, não é uma tarefa trivial no cuidado intensivo a pacientes gravemente enfermos. $\mathrm{O}$ controle 
glicêmico, realizado pela equipe de enfermagem, envolve a monitorização da glicemia a cada hora, a aplicação de protocolos frente aos resultados, a necessidade de ajustes na velocidade de infusão da insulina IV e o suporte nutricional adequado. ${ }^{29} \mathrm{Fa}-$ lhas ocorridas neste processo assistencial acarretam dano ao paciente, que podem se manifestar desde uma sudorese até coma e morte. ${ }^{26-27}$

O controle glicêmico efetivo é fundamental para evitar/reduzir a ocorrência de EAM. Além dos fatores relacionados à gravidade do paciente, deve-se considerar as condições ligadas à estrutura do serviço e aos processos adotados na unidade, aspectos que envolvem desde a ausência/deficiência de procedimentos normatizados, capacitação da equipe de enfermagem, a técnica de coleta de sangue para aferição da glicemia, disponibilidade de aparelho de glicosímetro portátil, atraso nas aferições da glicemia, interrupção de nutrição enteral ou parenteral, o registro clínico inadequado e a falha de comunicação entre as equipes de saúde. ${ }^{9,29-31}$ Falhas na estrutura e no processo podem ocasionar resultados adversos ou indesejáveis, como morte, dano, doença ou insatisfação do paciente. ${ }^{32}$

Erros envolvendo o uso de insulina estão associados à falha na interpretação de abreviaturas, administração de dose e velocidade de infusão inadequada, omissão de dose, troca por frasco-ampola com outros medicamentos, administração ao paciente incorreto e interações com outros medicamentos ou com a dieta. ${ }^{28}$

O uso de protocolo assistencial para o controle glicêmico visa manter a glicemia dentro da faixa selecionada, evitar/reduzir a ocorrência de hipoglicemia e fornecer instruções específicas para o tratamento imediato de hipoglicemia, caso ocorra. No entanto, protocolos que apresentam baixa efetividade aumentam o risco de complicações, principalmente EAM. ${ }^{9}$

Entre os pacientes que apresentaram hipoglicemia relacionada ao uso de insulina, verificou-se uma grande variabilidade nos níveis glicêmicos (16 a $449 \mathrm{mg} / \mathrm{dL}$ ), podendo representar uma baixa efetividade do protocolo de controle glicêmico. A variabilidade glicêmica aumenta o risco de hipoglicemia e favorece as complicações crônicas do diabetes. ${ }^{11}$

\section{CONCLUSÃO}

A ocorrência de hipoglicemia é um problema comum em pacientes hospitalizados, particularmente, naqueles gravemente enfermos e impacta na mortalidade na terapia intensiva. A detecção da mag- nitude de hipoglicemia grave e dos fatores preditivos para sua ocorrência (presença de doença hepática, sepse e EA) favorece a implantação de medidas que visam melhorar os processos de enfermagem no manejo do controle glicêmico de pacientes hospitalizados, a qualidade da assistência e a segurança.

A hipoglicemia relacionada ao uso de insulina regular e NPH é um dano desnecessário provocado ao paciente decorrente da assistência em saúde. A implantação de um protocolo assistencial para o controle glicêmico na terapia intensiva é fundamental para manter a glicemia na faixa adequada e para evitar e tratar a ocorrência de hipoglicemia. Este protocolo deve abranger o manejo do controle glicêmico em pacientes diabéticos e não diabéticos, em jejum prolongado ou em outras situações clínicas de risco de hipoglicemia.

Compreender adequadamente o processo subjacente à ocorrência de eventos indesejáveis e prejudiciais ao paciente, sua família, ao hospital e à sociedade requer a incorporação de estratégias de avaliação no cotidiano assistencial. É possível implementar medidas que, longe de cunho punitivo, orientam padrões baseados na melhor evidência e fortalecem ações que minimizam a ocorrência de falhas.

Analisar as causas, não relacionadas aos medicamentos, mas associadas à ocorrência de hipoglicemia na UTI permitirá a detecção de novos eventos adversos, baseados em outra racionalidade. Novas pesquisas precisam avançar na avaliação de aspectos relacionados à estrutura física da unidade intensiva, a disponibilidade de materiais/equipamentos e de recursos humanos e analisar essa associação com a hipoglicemia.

\section{Agradecimentos}

À CAPES (processo $n^{\circ}$ BEX 15930/12-2) e à FAPERJ (processo n. E-26/103.052/2012) pelo apoio financeiro.

\section{REFERÊNCIAS}

1. Marik PE, Preiser J-C. Toward understanding tight glycemic control in the ICU: a systematic review and metaanalysis. CHEST J [Internet]. 2010 [cited 2016 Ago 25]; 137(3):544-51. Available from: http:/ / www.ncbi. nlm.nih.gov/pubmed/20018803

2. Boucai L, Southern WN, Zonszein J. Hypoglycemiaassociated Mortality Is Not Drug-associated but Linked to Comorbidities. Am J Med [Internet]. 2011 Nov [cited 2016 Ago 25]; 124(11):1028-35. Available from: https:/ / www.ncbi.nlm.nih.gov/pmc/articles / PMC3200530/pdf/nihms313990.pdf 
3. Nirantharakumar K, Marshall T, Hodson J, Narendran P, Deeks J, Coleman JJ, et al. Hypoglycemia in NonDiabetic In-Patients: Clinical or Criminal? Sesti G, editor. PLoS ONE [Internet]. 2012 Jul 2 [cited 2016 Ago 25]; 7(7):e40384. Available from: http://journals.plos. org/plosone/article/asset?id=10.1371\%2Fjournal. pone.0040384.PDF

4. The NICE-SUGAR Study Investigators. Hypoglycemia and Risk of Death in Critically Ill Patients. N Engl J Med [Internet]. 2012 Sep 20 [cited 2016 Ago 25]; 367(12):1108-18. Available from: https:// www.researchgate.net/publication/230883930_ Hypoglycemia_and_Risk_of_Death_in_Critically_ Ill_Patients

5. Todi S, Bhattacharya M. Glycemic variability and outcome in critically ill. Indian J Crit Care Med PeerRev Off Publ Indian Soc Crit Care Med [Internet]. 2014 May [cited 2016 Ago 25]; 18(5):285-90. Available from: https:/ / www.ncbi.nlm.nih.gov/pmc/articles/ PMC4047689/

6. on behalf of the CGAO-REA Study Group, Kalfon P, Le Manach Y, Ichai C, Bréchot N, Cinotti R, et al. Severe and multiple hypoglycemic episodes are associated with increased risk of death in ICU patients. Crit Care [Internet]. 2015 Dec [cited 2016 Ago 25]; 19(1). Available from: https://www.ncbi.nlm.nih. gov/pmc/articles/PMC4470320/pdf/13054_2015_ Article_851.pdf

7. Hermanides J, Bosman RJ, Vriesendorp TM, Dotsch R, Rosendaal FR, Zandstra DF, et al. Hypoglycemia is associated with intensive care unit mortality. Crit Care Med [Internet]. 2010 Jun [cited 2016 Ago 27]; 38(6):1430-4. Available from: http://www.ncbi.nlm. nih.gov/pubmed/20386307

8. DeVries JH. Glucose Variability: Where It Is Important and How to Measure It. Diabetes [Internet]. 2013 May 1 [cited 2016 Ago 25]; 62(5):1405-8. Available from: http:/ / diabetes.diabetesjournals.org/content/ diabetes/62/5/1405.full.pdf

9. Kelly JL. Continuous Insulin Infusion: When, Where, and How? Diabetes Spectr [Internet]. 2014 [cited 2016 Ago 25]; 27(3):218-23. Available from: http:// www.ncbi.nlm.nih.gov/pmc/articles/PMC4523736/ pdf/218.pdf

10. Zhu W, Jiang L, Jiang S, Ma Y, Zhang M. Real-time continuous glucose monitoring versus conventional glucose monitoring in critically ill patients: a systematic review study protocol. BMJ Open [Internet]. 2015 [cited 2016 Ago 25]; 5(1):e006579. Available from: http://bmjopen.bmj.com/content/5/1/e006579. full.pdf + html

11. Suh S, Kim JH. Glycemic Variability: How Do We Measure It and Why Is It Important? Diabetes Metab J [Internet]. 2015 [cited 2016 Ago 25]; 39(4):273. Available from: http://www.ncbi.nlm.nih.gov/pmc/ articles/PMC4543190/pdf/dmj-39-273.pdf

12. Boom DT, Sechterberger MK, Rijkenberg S, Kreder S,
Bosman RJ, Wester J, et al. Insulin treatment guided by subcutaneous continuous glucose monitoring compared to frequent point-of-care measurement in critically ill patients: a randomized controlled trial. Crit Care [Internet]. 2014 [cited 2016 Ago 25]; 18(4):453. Available from: https://www.ncbi.nlm.nih.gov/ pmc/articles/PMC4161875/

13. Dias MAE, Martins M, Navarro N. Adverse outcome screening in hospitalizations of the Brazilian Unified Health System. Rev Saúde Pública [Internet]. 2012 Aug [cited 2016 Aug 29]; 46(4):719-29. Available from: http://www.scielo.br/scielo.php?script=sci arttext\&pid=S0034-9102012000400017\&lng=en.

14. Institute for Healthcare Improvement. IHI Global Trigger Tool for Measuring Adverse Events [Internet]. IHI Innovation Series white paper. Cambridge, Massachusettts: Institute for Healthcare Improvement, 2009 [cited 2016 Ago 27]. Available from: http:// www.ihi.org/resources/pages/ihiwhitepapers/ ihiglobaltriggertoolwhitepaper.aspx

15. Runciman W, Hibbert $P$, Thomson R, Van Der Schaaf $\mathrm{T}$, Sherman H, Lewalle P. Towards an International Classification for Patient Safety: key concepts and terms. Int J Qual Health Care [Internet]. 2009 Feb 1 [cited 2016 Ago 25]; 21(1):18-26. Available from: https:/ / www.ncbi.nlm.nih.gov/pmc/articles / PMC2638755/pdf/mzn057.pdf

16. Rozenfeld S, Chaves SMC, Reis LGC, Martins M, Travassos C, Mendes W et al. Efeitos adversos a medicamentos em um hospital público: estudo piloto. Rev Saúde Pública [Internet]. 2009 [cited 2017 Mar 25]; 43(5):887-90. Available from: http:/ / www.scielo.br/ pdf/rsp/v47n6/0034-8910-rsp-47-06-01102.pdf

17. Reis AMM, Cassiani SHDB. Adverse drug events in an intensive care unit of a university hospital. Eur J Clin Pharmacol [Internet]. 2011 [cited 2017 Mar 27]; 67:625-32. Available from: https://www.ncbi.nlm. nih.gov/pubmed/21246350

18. Roque KE, Melo ECP. Avaliação dos eventos adversos a medicamentos no contexto hospitalar. Esc. Anna Nery [Internet]. 2012 Mar [cited 2017 Mar 27] ; 16(1): 121-27. Available from: http://www. scielo.br/scielo.php?script=sci_arttext\&pid=S141481452012000100016\&lng=en.

19. Giordani F, Rozenfeld S, Martins M. Adverse drug events identified by triggers at a teaching hospital in Brazil. BMC Pharmacol Toxicol [Internet]. 2014 Dec [cited 2017 Mar 27]; 15:71. Available from: https:/ / bmcpharmacoltoxicol.biomedcentral.com/ articles/10.1186/2050-6511-15-71

20. Roque KE, Tonini T, Melo ECP. Eventos adversos na unidade de terapia intensiva: impacto na mortalidade e no tempo de internação em um estudo prospectivo. Cad. Saúde Pública [Internet]. 2016 Oct [cited 2017 Mar 26] ; 32(10): e00081815. Available from: http:/ /www. scielo.br/scielo.php?script=sci_arttext\&pid=S0102311X2016001005001\&lng=en. 
21. Naranjo CA, Busto U, Sellers EM, Sandor P, Ruiz I, Roberts EA, et al. A method for estimating the probability of adverse drug reactions. Clin Pharmacol Ther [Internet]. 1981 Aug 1 [cited 2016 Ago 27]; 30(2):239-45. Available from: http://onlinelibrary. wiley.com/doi/10.1038/clpt.1981.154/pdf

22. Quan $H$, Sundararajan $V$, Halfon $P$, Fong $A$, Burnand $\mathrm{B}$, Luthi J-C, et al. Coding algorithms for defining comorbidities in ICD-9-CM and ICD-10 administrative data. Med Care [Internet]. 2005 Nov [cited 2016 Ago 25]; 43(11):1130-9. Available from: http:/ / citeseerx. ist.psu.edu/viewdoc/download?doi=10.1.1.466.475 $6 \&$ rep $=$ rep $1 \&$ type $=$ pdf

23. Garg R, Hurwitz S, Turchin A, Trivedi A. Hypoglycemia, With or Without Insulin Therapy, Is Associated With Increased Mortality Among Hospitalized Patients. Diabetes Care [Internet]. 2013 May 120 [cited 2016 Ago 25]; 36(5):1107-10. Available from: https://www.ncbi.nlm.nih.gov/pmc/articles/ PMC3631882/pdf/1107.pdf

24. Raurell TM, del LSC, AlmirallSD, Catalan IRM, Nicolás AJM. The optimal blood glucose target in critically ill patient: comparison of two intensive insulin therapy protocols. Med Clin (Barc) [Internet]. 2014 Mar [cited 2017 Mar 27]; 142(5):192-9. Available from: https:/ / www.ncbi.nlm.nih.gov/pubmed/23490488

25. Brutsaert E, Carey M, Zonszein J. The clinical impact of inpatient hypoglycemia. J Diabetes Complications [Internet]. 2014 Jul-Aug [cited 2017 Mar 27]; 28(4):56572. Available from: https://www.ncbi.nlm.nih.gov/ pubmed/24685363

26. Instituto para as Práticas Seguras no Uso de Medicamentos. Medicamentos Potencialmente Perigosos de uso hospitalar e ambulatorial - Listas atualizadas 2015. Boletim ISMP Brasil [Internet]. 2015 [cited 2017 Mar 27]; 1(2):1-2. Available from: http://www.ismp-brasil.org/site/wp-content/ uploads/2015/12/V4N3.pdf
27. Instituto para as Práticas Seguras no Uso de Medicamentos. Erros de medicação, riscos e práticas seguras na Terapia com insulinas. Boletim ISMP Brasil [Internet]. 2012 [cited 2016 Ago 29]; 1(2):1-2. Available from: http://www.ismp-brasil.org/site/wp-content/ uploads/2015/07/V1N2.pdf

28. Kansagara D, Fu R, Freeman M, Wolf F, Helfand M. Intensive insulin therapy in hospitalized patients: a systematic review. Ann Intern Med [Internet]. 2011 [cited 2016 Ago 25]; 154(4):268-82. Available from: http:/ / annals.org/article.aspx?articleid=746819

29. Paixão $C T$, Silva LD da, Doerzapff PB, Granadeiro RM de A, Farias RL de A, Santos SS. Risk factors for hypoglycemia in patients using continuous infusion of insulin in intensive care. ABCS Health Sci [Internet]. 2014 Dec 12 [cited 2016 Aug 29]; 39(3). Available from: https://www.portalnepas.org.br/abcshs/article/ view/655/654

30. Minuzzi AP, Salum NC, Locks MOH. Assement of patient safety culture in intensive care from the health team's perspective. Texto Contexto Enferm. [Internet]. 2016 [cited 2017 Jun 03]; 25(2):e1610015. Available from: http://www.scielo.br/scielo.php?script=sci_ arttext\&pid=S0104-07072016000200313\&lng=pt.

31. Mello JF de, Barbosa SFF. Cultura de segurança do paciente em terapia intensiva: recomendações da enfermagem. Texto Contexto Enferm. [Internet]. 2013 Dez [cited 2017 Jun 03]; 22(4):1124-33. Available from: http://www.scielo.br/scielo.php?script=sci arttext\&pid=S0104-07072013000400031\&lng=pt.

32. Silva LD da, Camerini FG. Analisys the intravenous medication administration in sentinel network hospital. Texto Contexto Enferm [Internet]. 2012 Set [cited 2016 Ago 29]; 21(3):633-41. Available from: http://www.scielo.br/scielo.php?script=sci arttext\&pid=S0104-07072012000300019\&lng=en.
Correspondência: Keroulay Estebanez Roque Hospital Universitário Clementino Fraga Filho, Universidade Federal do Rio de Janeiro.

Rua Rodolpho Paulo Rocco, 255, Sala 5E 17.

21941-913 - Cidade Universitária - Ilha do Fundão. Rio de Janeiro, RJ, Brasil

E-mail: keroulay@gmail.com
Recebido: 04 de setembro de 2016 Aprovado: 10 de agosto de 2017

This is an Open Access article distributed under the terms of the Creative Commons (CC BY). 